From the Massachusetts General Hospital; Beth Israel Deaconess Medical Center; and the Dana-Farber Cancer Institute, Boston, MA.

Submitted August 8, 2005; accepted February 2, 2006

Supported by Pfizer Inc

Authors' disclosures of potential conflicts of interest and author contributions are found at the end of this article.

Address reprint requests to Matthew Smith, MD, PhD, Massachusetts General Hospital Cancer Center, Yawkey 7038 55 Fruit St, Boston MA 02114; e-mail: smith.matthew@mgh.harvard.edu.

(C) 2006 by American Society of Clinical Oncology

0732-183X/06/2418-2723/\$20.00 DOI: $10.1200 / J C O .2005 .03 .7804$

\title{
Celecoxib Versus Placebo for Men With Prostate Cancer and a Rising Serum Prostate-Specific Antigen After Radical Prostatectomy and/or Radiation Therapy
}

Matthew R. Smith, Judith Manola, Donald S. Kaufman, William K. Oh, Glenn J. Bubley, and Philip W. Kantoff

\section{$\begin{array}{lllllllll}A & B & S & T & R & A & C & T\end{array}$}

\section{Purpose}

To assess the biologic activity of celecoxib, a selective cyclooxygenase- 2 inhibitor, in men with recurrent prostate cancer using change in prostate-specific antigen (PSA) doubling time (PSADT) as the primary outcome variable.

\section{Patients and Methods}

Participants had histologically confirmed prostate cancer, no recent hormone therapy, rising serum PSA after radical prostatectomy and/or radiation therapy, and no radiographic evidence of metastases. Patients were randomly assigned to celecoxib (400 mg by mouth twice daily) or placebo. Treatment continued until disease progression or until adverse effects stopped treatment. A positive outcome was defined as post-treatment PSADT of more than $200 \%$ baseline PSADT with no new metastases.

\section{Results}

The study was terminated early after information about the cardiovascular safety of celecoxib prompted review of ongoing clinical studies. Before discontinuation of the study, 78 men were assigned randomly to either celecoxib or placebo. Eight (20\%) of 40 men in the placebo group and $15(40 \%)$ of 38 men in the celecoxib group had post-treatment PSADT of more than $200 \%$ of baseline PSADT with no new metastases $(P=.08)$. Mean PSA velocity increased by $3.0 \%$ for the placebo group and decreased by $3.4 \%$ for the celecoxib group $(P=.02)$.

\section{Conclusion}

Although the primary efficacy objective was not met, this study provides some evidence for biologic activity of celecoxib in prostate cancer. Compared with placebo, celecoxib significantly decreased mean PSA velocity and tended to increase the proportion of men who doubled their PSADT

\section{J Clin Oncol 24:2723-2728. (C) 2006 by American Society of Clinical Oncology}

\section{INTRODUCTION}

Prostate cancer is the most common solid tumor in men worldwide. In 2005, there were approximately 232,090 new cases of prostate cancer and 30,350 prostate cancer deaths in the United States. ${ }^{1}$ Approximately $80 \%$ of new cases are clinically localized, and most men with these early-stage prostate cancers are treated with radical prostatectomy or radiation therapy. Approximately one third to one half of patients experience disease recurrence after definitive local treatment.

Rising serum prostate-specific antigen (PSA) levels after radical prostatectomy or radiation therapy provides an early indication of recurrent disease. A serial rise of serum PSA levels after surgery or radiation therapy, however, typically predates by many years clinically or radiographically detectable metastatic disease without additional treatment. In a retrospective series of men with a rising PSA after radical prostatectomy, for example, the median time to metastasis was 8 years from the time of PSA elevation. ${ }^{2}$

Increases in serum PSA following radical prostatectomy or radiation therapy follow an exponential growth curve and the relationship between log PSA and time is linear. ${ }^{2-4}$ Log-slope PSA, PSA doubling time (PSADT), Gleason grade, and the interval between local treatment and biochemical progression predict the probability of survival and the time to development of distant metastatic disease. The PSADT or the equivalent log-slope PSA are better predictors of the probability and time to clinical recurrence than preoperative PSA levels, the interval 
between local treatment and rising PSA, Gleason grade, or pathologic stage. $^{2,3}$ In men with a rising PSA after radical prostatectomy, a PSADT shorter than the median value of 10 months is the most significant predictor for progression to metastatic disease found by Pound et al. ${ }^{2}$ Similarly, a short post-treatment PSADT following radiation therapy predicts progression to metastatic disease. ${ }^{3,4}$ PSA velocity in men with a rising PSA following definitive local treatment is significantly related to survival. ${ }^{5,6}$

Men with an isolated PSA recurrence after local treatment represent an ideal population for the evaluation of novel therapies based on minimal disease state, indolent natural history, and preference to avoid the adverse effects of hormone therapy. The evaluation of novel agents in this setting, however, is hampered by the lack of convenient validated end points. Overall or progression-free survival end points are impractical because of the long interval between an initial PSA increase and development of metastases. In addition, PSA response criteria commonly used in androgen-independent prostate cancer ${ }^{7}$ may neglect biologically and potentially clinically meaningful activity, particularly with cytostatic agents. Changes in PSADT may be more sensitive to detect biologic activity than traditional PSA response criteria, although PSADT has not been adequately evaluated as a clinical trial end point.

Cyclooxygenase (COX) enzymes catalyze the synthesis of prostaglandins from arachidonic acid. The COX-1 isoform is constitutively expressed in most tissues. In contrast, COX-2 is not expressed in most normal tissues but is rapidly induced by a variety of inflammatory and mitogenic stimuli. COX-2 has been implicated in the pathogenesis of a variety of human malignancies. Preclinical and clinical evidence suggest that COX-2 is an attractive target for the treatment or prevention of prostate cancer. Some but not all studies suggest that COX-2 is overexpressed in the primary human prostate cancers and human prostate cancer cell lines. ${ }^{8-12}$ Selective COX-2 inhibitors induce apoptosis of human prostate cancer cells in vitro and in vivo. ${ }^{13,14}$ Lastly, treatment with nonspecific COX-2 inhibitors is associated with a lower risk of prostate cancer. ${ }^{15}$

Celecoxib (Celebrex; Pfizer Inc, New York, NY) is a selective COX-2 inhibitor indicated for osteoarthritis, rheumatoid arthritis, acute pain, primary dysmenorrhea, and familial adenomatous polyposis. In this study, we prospectively compared the effects of celecoxib and placebo in men with a rising PSA after radical prostatectomy and/or radiation therapy. We evaluated changes in PSADT as a screen for biologic and clinical activity.

\section{PATIENTS AND METHODS}

\section{Study Participants}

Study participants were recruited at the Dana-Farber Cancer Institute (Boston, MA), Massachusetts General Hospital (Boston, MA), Beth Israel Deaconess Medical Center (Boston, MA), The University of Texas M.D. Anderson Cancer Center (Houston, TX), Hartford Hospital (Hartford, CT), Lowell General Hospital (Lowell, MA), and the University of Michigan (Ann Arbor, MI) between October 2002 and December 2004. Participants had histologically confirmed adenocarcinoma of the prostate, biochemical disease progression after radical prostatectomy and/or radiation therapy (external-beam radiation therapy and/or brachytherapy), and no radiographic evidence of metastases. Biochemical progression was defined as three rises in PSA levels, with each PSA determination at least 4 weeks apart, and each PSA value $\geq 0.2 \mathrm{ng} / \mathrm{mL}$. Men with history of radical prostatectomy were required to have baseline PSA $\geq 1 \mathrm{ng} / \mathrm{mL}$. Men treated with primary radiation therapy were required to have baseline PSA $\geq 3$ $\mathrm{ng} / \mathrm{mL}$ and postradiation nadir greater than $150 \%$. Men with baseline PSADT $\leq 6$ months or $\geq 24$ months were excluded. Men with previous neoadjuvant or adjuvant hormone therapy were included if the interval between completion of hormone therapy and study entry was greater than 1 year. Men who received radiation therapy within 6 months of study entry, who received any treatment with chemotherapy for prostate cancer, or who received previous hormonal therapy for recurrent prostate cancer were excluded. Participants receiving low-dose aspirin ( $\leq 325 \mathrm{mg}$ daily) were included. Participants who received treatment with a nonsteroidal anti-inflammatory drug or COX-2 inhibitor within 8 weeks of study entry were excluded. Participants with Cancer and Leukemia Group B performance status $\geq 2$, allergy to nonsteroidal anti-inflammatory drugs, history of gastrointestinal ulcers or bleeding within 12 months of study entry, myocardial infarction within 12 months, or current treatment with fluconazole, lithium, or warfarin were excluded. Subjects with WBC less than 3,000 , absolute neutrophil count less than 1,500, hemoglobin count less than $10 \mathrm{~g} / \mathrm{dL}$, platelet count less than 100,000, creatinine level of more than $1.5 \mathrm{mg} / \mathrm{mL}$, or serum ALT, AST, or total bilirubin $\geq 1.5 \times$ the upper limit of normal were also excluded.

\section{Study Design}

The study was a prospective, randomized, placebo-controlled clinical trial with optional cross-over at 6 months. Participants were stratified according to history of prostatectomy (yes $v$ no), current use of cardioprotective doses of aspirin (yes $v$ no), and baseline PSADT ( $<10$ months $v \geq 10$ months). At a baseline visit, participants underwent a physical examination, radionuclide bone scan, abdominal and pelvic computed tomography scans, ECG, complete blood count, routine serum chemistries, and serum PSA. Eligible participants were randomly assigned to treatment with celecoxib (400 mg by mouth twice daily) or placebo for 6 months. No dose or schedule modifications were permitted. Study participants and study personnel were blinded to treatment assignments. Other therapies for prostate cancer were not permitted during the study. Serum PSA was measured every 4 weeks.

Study participants continued blinded treatment for 6 months. Treatment was discontinued early for any participants with an expected Common Toxicity Criteria (CTC) grade 3 or greater toxicity, unexpected grade 3 or greater toxicity that was definitely or probably related to study drug, or progressive disease (defined in Study Outcomes section). Participants who discontinued treatment because of toxicity were withdrawn from the study. Treatment assignments were unblinded for participants who completed 6 months of treatment or discontinued early because of progressive disease. Participants assigned to placebo were eligible to cross-over to open-label treatment with celecoxib for six months. Open-label treatment was discontinued early for any subject with an expected CTC grade 3 or greater toxicity, unexpected grade 3 or greater toxicity that was definitely or probably related to study drug, or progressive disease.

The institutional review boards of each of the participating institutions approved the study. All study participants gave written informed consent. The study sponsors played no role in the study 
design, in collection, analysis and interpretation of data, or in the writing of this report.

\section{Study Outcomes}

PSADT was calculated by natural log of 2 (0.693) divided by the slope of the relationship between the natural log of PSA and time. ${ }^{2}$ Slope was calculated by linear regression. The baseline (pretreatment) PSADT was calculated using the three PSA level increases required for study entry, all other PSA values obtained during the interval between the first and third PSA level increase, and the PSA value immediately preceding the first PSA increase. The post-treatment PSADT was calculated using PSA measurements obtained at baseline and monthly for the first 6 months of treatment. For patients who discontinued treatment before 6 months, all available PSA measurements before discontinuation of treatment were used to calculate PSADT. All participants with either progressive disease or at least one post-treatment PSA measurement were considered assessable.

A positive PSADT outcome was defined as either a posttreatment PSADT of more than $200 \%$ of the baseline PSADT or a negative post-treatment PSADT (declining PSA) with no new metastases. Disease progression was defined as new evidence of metastatic disease, or PSA $\geq 200 \%$ of baseline value.

\section{Statistical Analyses}

The primary study objective was to compare the proportion of men with a positive PSADT outcome between the two groups. The study was designed to distinguish between a $65 \%$ positive outcome rate for celecoxib-treated patients and a $35 \%$ positive outcome rate for placebo-treated participants. With a target accrual of 140 participants (65 assessable participants and five unassessable participants per arm), the design provided $90 \%$ power using a two-sided Fisher's exact test with type I error of $5 \%$.

Fisher's exact test was used to compare the proportion of men with a positive PSADT outcome between the groups. The Wilcoxon rank sum test was used to compare PSA slopes and PSA doubling times between groups. This test was also used to compare posttreatment changes in PSA slope between the groups, and to compare changes in PSA velocity between the blinded and open-label treatment period for participants assigned to placebo.

\section{RESULTS}

\section{Participant Characteristics}

In December 2004, the study was discontinued before the accrual goal of 140 participants, after information about the cardiovascular safety of celecoxib ${ }^{16}$ prompted review of ongoing clinical studies. Before the study was discontinued, 78 men were randomly assigned to either celecoxib or placebo. Baseline characteristics including age, prior prostatectomy, and PSA were similar between the groups (Table 1). Mean baseline PSADT was 12.7 months(SD, 6.0 months) in the placebo group and 12.1 months (SD, 4.5 months) in the celecoxib group. Fourteen men (eight assigned to placebo, six assigned to celecoxib) completed less than 6 months of treatment because of early discontinuation of the study; these participants were included in the analyses.

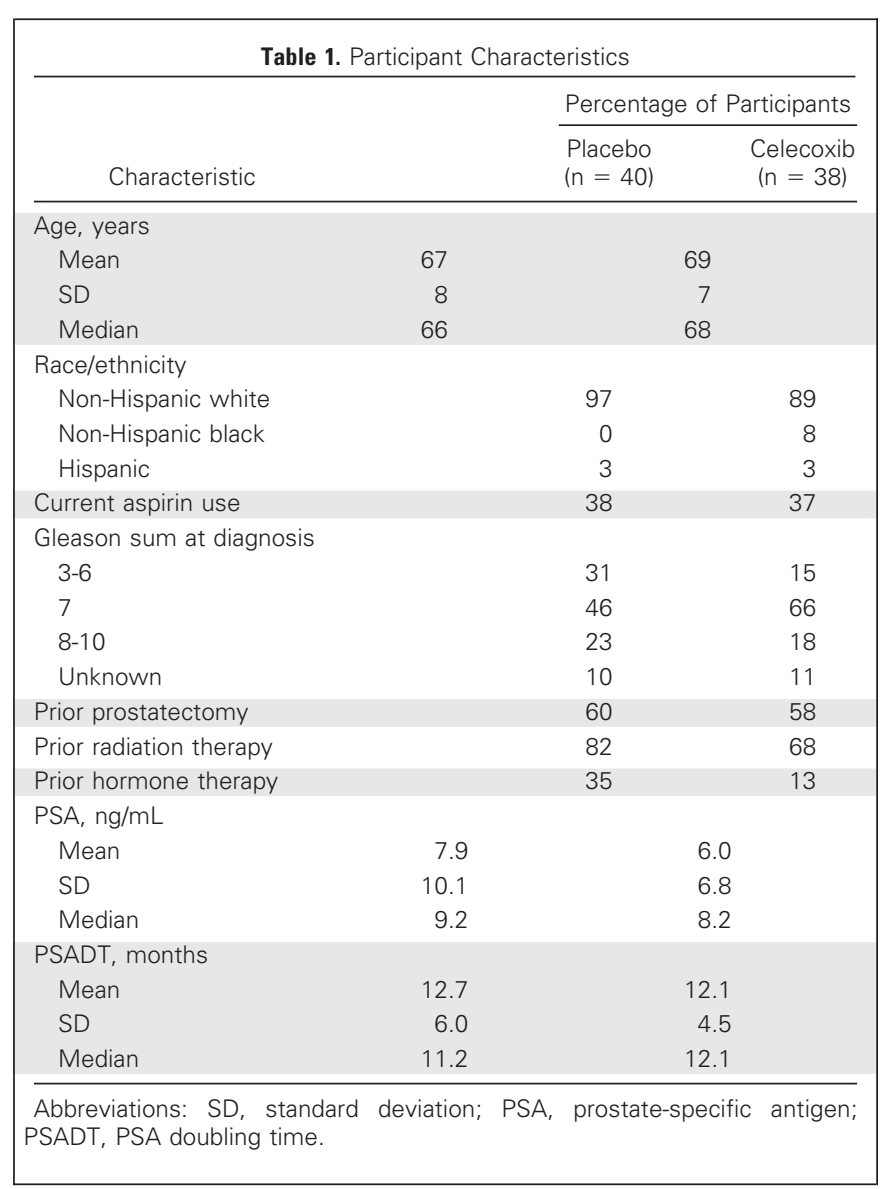

\section{PSA Outcomes}

Figure 1 shows log-PSA measurements during the pretreatment, randomized, and open-label periods for men randomly assigned to placebo and celecoxib.

Eight (20\%) of 40 men in the in the placebo group and 15 (40\%) of 38 men in the celecoxib group had post-treatment PSADT of more than $200 \%$ of baseline PSADT with no new metastases $(P=.08$; Table $2)$. Figure 2 illustrates the relationships between baseline and posttreatment PSADT for each group. The mean change in PSA velocity differed significantly between the groups. Mean PSA velocity increased by $3.0 \%$ for the placebo group and decreased by $3.4 \%$ for the celecoxib group ( $P=.02$ for between-group comparison).

Twenty-nine men in the placebo group crossed over to openlabel treatment with celecoxib. Ten of these men (35\%) had PSADT during open-label celecoxib treatment that was more than $200 \%$ of PSADT during placebo treatment. Mean PSA velocity decreased by $7.4 \%$ after cross over to open-label treatment, although this change was not statistically significant $(P=.59)$. Figure 3 shows the relationships between PSADT during placebo treatment and PSADT during open-label celecoxib treatment.

\section{Adverse Events}

There were no deaths during the study. Serious adverse events (all grade 3) were reported in four participants in the placebo group (supraventricular arrhythmia, bipolar disorder, erectile dysfunction, incontinence, and pleural effusion) and five patients in the celecoxib group (erectile dysfunction, incontinence, hypertension, sinus 


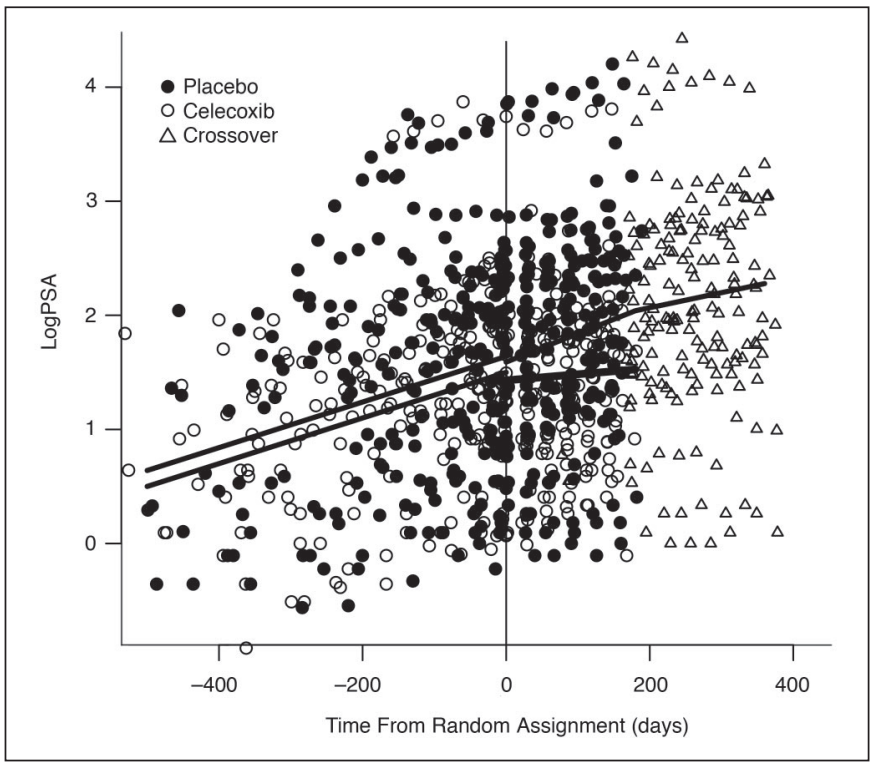

Fig 1. Log prostate-specific antigen (PSA) measurements during the pretreatment, randomized, and open-label periods for men randomly assigned to placebo (thin line) and celecoxib (thick line).

bradycardia, rash, abnormal stress test, and the detection of pulmonary nodules). These adverse events led to study discontinuation for one participant in the placebo group (bipolar disorder) and three participants in the celecoxib group (one for rash, one for abnormal stress test and pulmonary nodules, and one for hypertension and bradycardia).

\section{DISCUSSION}

We compared the effects of celecoxib and placebo on the change in PSA kinetics in men with a rising PSA after radical prostatectomy and/or radiation therapy. Although the primary study outcome was negative, there was some evidence for biologic activity of celecoxib in prostate cancer. First, more men in the celecoxib group doubled their PSADT than in the placebo group (40\% v 20\%; $P=.08)$. Second, mean post-treatment change in PSA velocity differed significantly between the placebo and celecoxib groups (3.0\% increase $v 3.4 \%$ decrease; $P=.02$ ). Third, 35\% of men in the placebo group doubled their PSADT after cross over to open-label celecoxib.

Our observations are consistent with the results of a recently reported pilot study of celecoxib in men with elevated serum PSA

\begin{tabular}{|c|c|c|c|c|c|}
\hline \multirow[b]{2}{*}{ Positive PSADT Outcome } & \multicolumn{2}{|l|}{ Placebo } & \multicolumn{2}{|c|}{ Celecoxib } & \multirow[b]{2}{*}{$P$} \\
\hline & $\begin{array}{c}\text { No. of } \\
\text { Participants }\end{array}$ & $\%$ & $\begin{array}{c}\text { No. of } \\
\text { Participants }\end{array}$ & $\%$ & \\
\hline Yes & 8 & 20 & 15 & 40 & .08 \\
\hline No & 32 & 80 & 23 & 60 & \\
\hline \multicolumn{6}{|c|}{$\begin{array}{l}\text { NOTE: Positive PSADT outcome was defined as post-treatment PSADT } \\
>200 \% \text { of baseline PSADT with no metastatic disease. } \\
\text { Abbreviation: PSADT, prostate-specific antigen doubling time. }\end{array}$} \\
\hline
\end{tabular}

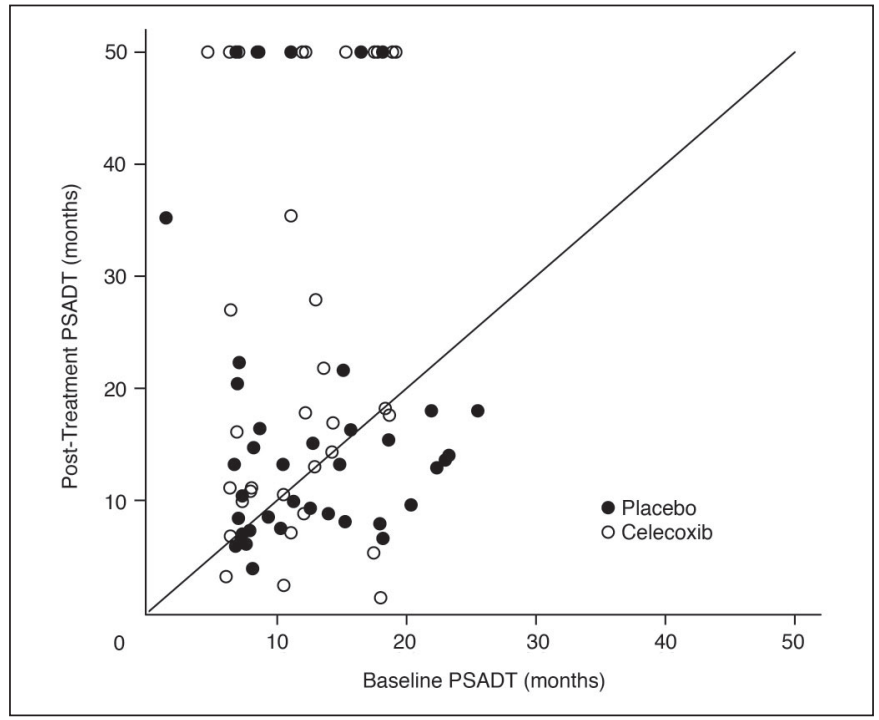

Fig 2. Relationship between baseline and post-treatment prostate-specific antigen doubling time (PSADT) for men in the placebo group (closed circles) and celecoxib group (open circles) during the blinded treatment period.

levels after radical prostatectomy or radiation therapy. ${ }^{17}$ At 6 months, five of 12 men had either stable or declining PSA. Mean PSA velocity decreased after celecoxib treatment.

Our results may have important implications about the use of PSADT or PSA velocity as a study end point. Twenty percent of placebo-treated participants had favorable outcomes, defined as posttreatment PSADT of more than $200 \%$ of baseline PSADT. In another randomized controlled trial of men with rising PSA after surgery or radiation therapy, $31 \%$ of placebo-treated participants had posttreatment PSADT of more than $200 \%$ of baseline PSADT. ${ }^{18}$ The high rate of positive PSADT outcomes may reflect the limited precision of PSADT determinations. Alternatively, the relatively high rate of PSADT prolongation may be related to the placebo effect. ${ }^{19}$ Mean

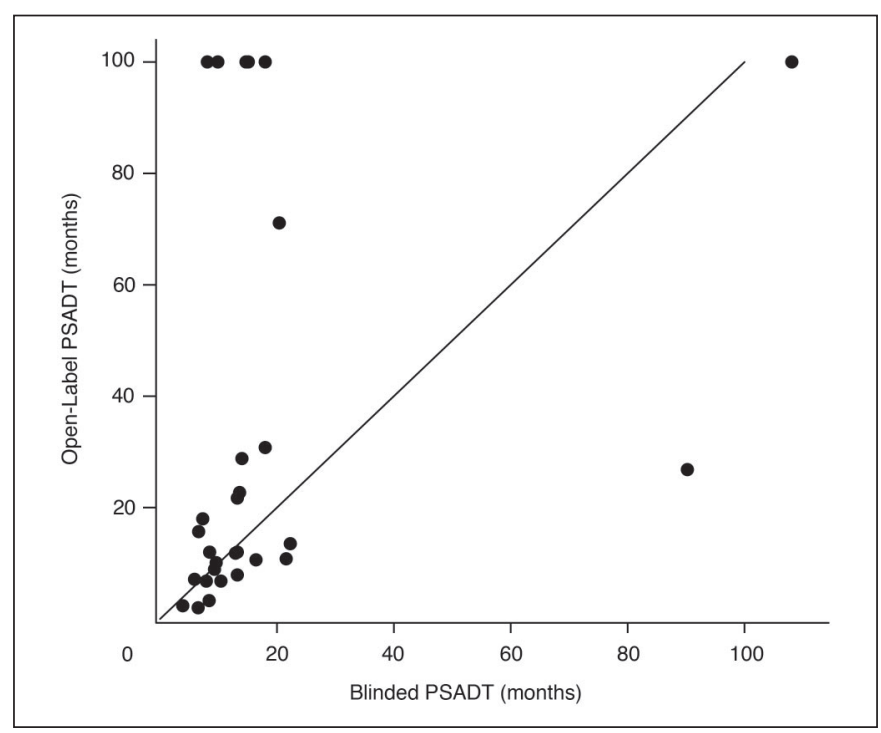

Fig 3. Relationships between prostate-specific antigen doubling time (PSADT) during placebo treatment and PSADT during open-label celecoxib treatment for the placebo group. 
post-treatment PSA slope did not change significantly from baseline in the placebo group, however, suggesting that the higher than expected rate of positive PSADT outcomes did not result from temporal changes in tumor growth rate. Larger studies to assess the relationship between PSA kinetics and clinical outcomes will be required to evaluate the utility of post-treatment changes in PSADT or PSA velocity in the clinical development of new drugs for prostate cancer.

Our study has limitations. The study was underpowered because of its early termination and incomplete accrual. Baseline PSADT was calculated using PSA values determined at irregular intervals, whereas post-treatment PSADT was calculated using monthly PSA values. For most participants, baseline PSADT was calculated using fewer PSA values than the post-treatment PSADT. Differences in the intervals between PSA measurements and in the number of PSA values may have contributed to the variability between baseline and posttreatment PSADT in the placebo group. We evaluated changes in PSADT as a screen for biologic and clinical activity and cannot exclude the possibility that celecoxib modulated PSA expression without altering growth or survival of prostate cancer cells. Larger studies with clinical end points would be necessary to assess whether celecoxib had meaningful clinical activity.

In summary, we observed evidence for modest biologic activity of celecoxib in men with rising PSA levels after radical prostatectomy and/or radiation therapy for prostate cancer. These results provide further rationale for the evaluation of COX-2 inhibitors in prostate cancer. When considering future clinical trials of COX-2 inhibitors for prostate cancer, however, the potential beneficial effects of COX-2 inhibition will have to be weighed against the possible increase in cardiovascular disease risk.

\section{REFERENCES}

1. Jemal A, Murray $T$, Ward $E$, et al: Cancer statistics, 2005. CA Cancer J Clin 55:10-30, 2005

2. Pound CR, Partin AW, Eisenberger MA, et al: Natural history of progression after PSA elevation following radical prostatectomy. JAMA 281:15911597, 1999

3. Patel A, Dorey F, Franklin J, et al: Recurrence patterns after radical retropubic prostatectomy: Clinical usefulness of prostate specific antigen doubling times and log slope prostate specific antigen. J Urol 158:1441-1445, 1997

4. Zagars GK, Pollack A: Kinetics of serum prostate-specific antigen after external beam radiation for clinically localized prostate cancer. Radiother Oncol 44:213-221, 1997

5. Vollmer RT, Dawson NA, Vogelzang NJ: The dynamics of prostate specific antigen in hormone refractory prostate carcinoma: An analysis of cancer and leukemia group B study 9181 of megestrol acetate. Cancer 83:1989-1994, 1998

6. D'Amico AV, Cote K, Loffredo M, et al: Determinants of prostate cancer-specific survival after radiation therapy for patients with clinically localized prostate cancer. J Clin Oncol 20:4567-4573, 2002
7. Bubley GJ, Carducci M, Dahut W, et al: Eligibility and response guidelines for phase II clinical trials in androgen-independent prostate cancer: Recommendations from the Prostate-Specific Antigen Working Group. J Clin Oncol 17:3461-3467, 1999

8. Gupta S, Srivastava M, Ahmad N, et al: Overexpression of cyclooxygenase-2 in human prostate adenocarcinoma. Prostate 42:73-78, 2000

9. Uotila $P$, Valve $E$, Martikainen $P$, et al: Increased expression of cyclooxygenase-2 and nitric oxide synthase-2 in human prostate cancer. Urol Res 29:23-28, 2001

10. Kirschenbaum A, Klausner AP, Lee $R$, et al: Expression of cyclooxygenase- 1 and cyclooxygenase-2 in the human prostate. Urology 56:671-676, 2000

11. Zha S, Gage WR, Sauvageot $J$, et al: Cyclooxygenase-2 is up-regulated in proliferative inflammatory atrophy of the prostate, but not in prostate carcinoma. Cancer Res 61:8617-8623, 2001

12. Shappell SB, Manning $S$, Boeglin WE, et al Alterations in lipoxygenase and cyclooxygenase-2 catalytic activity and mRNA expression in prostate carcinoma. Neoplasia 3:287-303, 2001

13. Kamijo $T$, Sato $T$, Nagatomi $Y$, et al: Induction of apoptosis by cyclooxygenase-2 inhibitors in prostate cancer cell lines. Int J Urol 8:S35-S39, 2001
14. Liu XH, Kirschenbaum A, Yao S, et al: Inhibition of cyclooxygenase-2 suppresses angiogenesis and the growth of prostate cancer in vivo. J Urol 164:820-825, 2000

15. Harris RE, Beebe-Donk J, Doss $H$, et al: Aspirin, ibuprofen, and other non-steroidal antiinflammatory drugs in cancer prevention: A critical review of non-selective COX-2 blockade (review). Oncol Rep 13:559-583, 2005

16. U.S. Food and Drug Administration.FDA Statement on the Halting of a Clinical Trial of the Cox-2 Inhibitor Celebrex. Rockville, MD, Food and Drug Administration, 2004 http://www.fda.gov/bbs/ topics/news/2004/new01144.html

17. Pruthi RS, Derksen JE, Moore D: A pilot study of use of the cyclooxygenase-2 inhibitor celecoxib in recurrent prostate cancer after definitive radiation therapy or radical prostatectomy. BJU Int 93:275278, 2004

18. Smith MR, Manola J, Kaufman DS, et al: Rosiglitazone versus placebo for men with prostate carcinoma and a rising serum prostate-specific antigen level after radical prostatectomy and/or radiation therapy. Cancer 101:1569-1574, 2004

19. Moyad MA: The placebo effect and randomized trials: Analysis of conventional medicine. Urol Clin North Am 29:125-133, ix-x, 2002

\section{Acknowledgment}

We thank the other investigators who participated in the study, including M. Dror Michaelson, Mary Ellen Taplin, Daniel George, Philip Febbo, Timothy Gilligan, Yoo-Joung Ko, Murat Anamur, Robert Siegel, Blair Ardman, Maha Hussein, David Smith, Kenneth Pienta, Jeri Kim, and Christopher Logothetis.

\section{Authors' Disclosures of Potential Conflicts of Interest}

Although all authors completed the disclosure declaration, the following authors or their immediate family members indicated a financial interest. No conflict exists for drugs or devices used in a study if they are not being evaluated as part of the investigation. For a detailed description of the disclosure categories, or for more information about ASCO's conflict of interest policy, please refer to the Author Disclosure Declaration and the Disclosures of Potential Conflicts of Interest section in Information for Contributors.

\begin{tabular}{|c|c|c|c|c|c|c|c|c|}
\hline Authors & Employment & Leadership & Consultant & Stock & Honoraria & Research Funds & Testimony & Other \\
\hline Glenn J. Bubley & & & & & & Pfizer (A) & & \\
\hline Philip W. Kantoff & & & Pfizer (A) & & & & & \\
\hline
\end{tabular}




\section{Author Contributions}

Conception and design: Matthew R. Smith, Judith Manola, Philip W. Kantoff

Provision of study materials or patients: Matthew R. Smith, Donald S. Kaufman, William K. Oh, Glenn J. Bubley, Philip W. Kantoff Collection and assembly of data: Matthew R. Smith, Judith Manola, Philip W. Kantoff

Data analysis and interpretation: Matthew R. Smith, Judith Manola, Philip W. Kantoff

Manuscript writing: Matthew R. Smith, Judith Manola, Donald S. Kaufman, William K. Oh, Glenn J. Bubley, Philip W. Kantoff

Final approval of manuscript: Matthew R. Smith, Judith Manola, Donald S. Kaufman, William K. Oh, Glenn J. Bubley, Philip W. Kantoff 